hältnis der Kanzler zu den Medien sowie ihre strukturellen und kommunikativen Maßnahmen im Rahmen der Öffentlichkeitsarbeit erklären könnten. Zu denken wäre hier etwa an die Ereignislage und die politische Situation auf Bundesebene, die Entwicklung des Mediensystems, die bestehenden Strukturen der Öffentlichkeitsarbeit sowie persönliche Faktoren.

Viertens finden sich auch einige sachliche Fehler und zumindest fragwürdige Aussagen. So wird beispielsweise behauptet, der Amtsbonus für die Kanzler in der Berichterstattung bestehe in ihrer besseren Bewertung (S. 47). Dies ist gerade nicht der Fall. Der Amtsbonus drückte sich bei früheren Wahlen in einer umfangreicheren und nicht in einer positiveren Berichterstattung aus. ${ }^{2}$ Kurios ist auch die unbelegte Feststellung, die Idee zur Gründung des Instituts für Demoskopie in Allensbach sei Anfang der 1950er Jahre in einem Kreis um Otto Lenz entstanden. Tatsächlich wurde das IfD Allensbach schon 1947 gegründet. $^{3}$

Insgesamt kann der Band von Lars Rosumek aus den genannten Gründen nicht überzeugen. Er macht aber zumindest deutlich, wo noch immer Lücken in der historischen Aufarbeitung des Verhältnisses zwischen Kanzlern und Medien bestehen.

Carsten Reinemann

2 Vgl. Jürgen Wilke / Carsten Reinemann, Die Normalisierung des Sonderfalls? Die Wahlkampfberichterstattung der Presse 2005 im Langzeitvergleich, in: Christina Holtz-Bacha (Hrsg.), Die Massenmedien im Wahlkampf. Die Bundestagswahl 2005, Wiesbaden 2006, S. 306 - 337.

3 Vgl. Elisabeth Noelle-Neumann, Die Erinnerungen, München 2006.

\title{
Herausforderungen für die Parteien in Deutschland: flott - und schnell - formuliert
}

Leif, Thomas: Angepasst und ausgebrannt: Die Parteien in der Nachwuchsfalle. Warum Deutschland der Stillstand droht, C. Bertelsmann Verlag, München 2009, 494 Seiten, $€ 22,95$.

Umschlaggestaltung und Titelgebung zeigen, dass Thomas Leif und der Bertelsmann-Verlag eine Neuauflage ihres Erfolges in den Buchhandlungen anstreben: 2006 erschien vom selben Autor „beraten und verkauft“ und wurde zum Sachbuch-Bestseller. Leif verteilte dort viele Schläge gegen Unternehmensberater, die aber oftmals am Ziel vorbeigingen: Verwendete Begriffe blieben unklar, Informationen wurden selektiv wahrgenommen und einseitig interpretiert - und das in einem selbstgerechten Ton, der auf der Welle der großen Kritik am Einsatz von Beratungsfirmen in Ministerien und insbesondere bei der Umstrukturierung der Bundesanstalt für Arbeit schwamm. Von der Politikwissenschaft ist das Buch daher zu Recht kaum beachtet worden.

Angesichts der fast identischen Gestaltung der Titelseite und der reißerischen Namensgebung ist daher bei ,angepasst und ausgebrannt“ zunächst Skepsis angebracht. Und tatsächlich findet man auch im Innern des Buches viele Ähnlichkeiten: flotte Formulierungen, Zitate aus internen „Geheimdokumenten“, reichlich Interviews mit Experten, die Reportage einer teilnehmenden Beobachterin - diesmal nicht zur Rekrutierung bei McKinsey, sondern beim CDU-Landesverband in Bremen. 
Auch der große Holzhammer wird wieder ausgepackt - nur trifft Leif jetzt doch häufiger den Nagel auf den Kopf. Das Buch reiht sich in die Parteienkritiken der vergangenen Jahrzehnte ein, die unter anderem durch Richard von Weizsäcker, Hans-Herbert von Arnim oder Jürgen Rüttgers formuliert wurden. Allerdings wird von Leif die für eine funktionierende parlamentarische Demokratie erforderliche Leistung von Parteien deutlich hervorgehoben: die übergreifende und längerfristige Integration und Bündelung von Interessen. Parteien erbringen - bei allen Schwächen - dazu einen wesentlichen Beitrag. Sie schaffen einen festen Rahmen im politischen Prozess und sorgen dafür, dass Entscheidungen nicht allein von aktuellen Stimmungen getragen aneinandergereiht werden.

So ist die Kritik weniger auf Parteien an sich, als auf die derzeit Handelnden gerichtet. Wenngleich Leif teilweise sehr allgemein von „den Parteiführungen“ spricht, wird auch deutlich, dass es sich bei Parteien nicht um monolithische oder strikt hierarchische Akteure handelt, sondern dass auf vielen Ebenen an Entscheidungen mitgewirkt wird; in Bezug auf die Kandidatenaufstellung weist er korrekt darauf hin, dass nur selten Einflussmöglichkeiten von jenseits der Orts- und Kreisverbände gegeben sind. Ausdrücklich bleibt kritische Distanz zu dem verbreiteten „Mythos Seiteneinsteiger“ gewahrt, und es werden die wesentlichen Sozialisations- und Trainingseffekte der innerparteilichen Tätigkeit gewürdigt (S. 425 ff.). Auch hebt der Autor hervor, dass neue Mitglieder meist mit offenen Armen empfangen und ihnen umfassende Mitwirkungsmöglichkeiten geboten werden, was der schon angesprochene Erfahrungsbericht von Tina Groll zur CDU in Bremen illustriert.

Die Krisendiagnose selbst ist indes nicht neu: Parteien verlieren seit den siebziger Jahren deutlich an Mitgliedern, und die Zahl der sich aktiv einbringenden ist gering. Während die Politikwissenschaft in den vergangenen Jahren eine Quote zwischen zehn und über 50 Prozent an Aktiven ermittelt hat, meint Leif unter Bezugnahme auf nicht näher genannte „Organisations-Insider“, dass man mit „zwei bis fünf Prozent ... sicher näher an der Realität sei“ (S. 210). Eine umfassende und nicht veröffentlichte Befragung von 10.000 Neumitgliedern der SPD habe im Jahr 2004 ermittelt, dass fast ein Drittel bereits bei Parteieintritt angibt, sich nicht aktiv beteiligen zu wollen (S. 219). Mittlerweile haben Parteien in ländlichen Regionen nicht selten Probleme, genug Kandidaten für zu besetzende eigene wie öffentliche Ämter und Mandate zu finden.

Leif hat eine ganze Reihe von internen Dokumenten zusammengetragen und ausgewertet, unter anderem Umfragen, Konzeptpapiere und Schulungsmaterialien, Organisationsgutachten für die SPD-Parteizentrale und die SPD-Geschäftsstellen in Nordrhein-Westfalen. Interessant ist die Darstellung der gezielten Versuche von Parteien, durch interne Fortbildungen und Mentoring-Programme junge Aktive zu fördern. So haben die Sozialdemokraten eine „SPD-Führungsakademie“ eingerichtet, und groß in Mode ist das „Mentoring“, das Nachwuchskräfte zum regelmäßigen Erfahrungsaustausch in direkten Kontakt mit etablierten Politikern bringen soll.

Die Stärke des Buches liegt darin, dass der Status quo der Parteiendemokratie in Deutschland abwechslungsreich beschrieben wird - allerdings sei dringend davor gewarnt, alle zitierten Aussagen für bare Münze zu nehmen. Der Forschungsstand ist teilweise unvollständig wiedergegeben, selbst die wenigen im Literaturverzeichnis enthaltenen Quellen wurden nicht umfassend ausgewertet (zum Beispiel zum Thema der Aktivitätsquote), und Angaben sind widersprüchlich oder unpräzise - so wird berichtet, dass im Jahr 2002 rund 60 Prozent der CDU-Direktkandidaten in Mitgliederversammlungen aufgestellt wurden (S. 64), und später heißt es: Wie oft Vollversammlungen tatsächlich angewendet wurden, 
sei „nicht zu ermitteln“ (S. 217). Ähnlich verwirrend ist, dass die Gesprächspartner in den abgedruckten Interviews darauf hinweisen, dass die Bedeutung von Referentenposten für den parteiinternen Aufstieg überbewertet sei (S. 92 f., S. 199 f., S. 289), Leif in seinem Fazit dann aber anhand von zwei Beispielen aus dem Bund und dem Land Hessen zu dem Ergebnis kommt, hier gelte das Prinzip „Aufstieg garantiert“ (S. 434).

Die Lösungsansätze und Empfehlungen für die Zukunft bieten wenig Neues und werden kaum reflektiert. Leif nennt die Einführung „direkter Demokratie-Partizipation “ durch Plebiszite oder Volksabstimmungen (S. 364 f.), das Aufstellen von Bürgerhaushalten (S. 368), den Einsatz von Vorwahlen zur Kandidatenaufstellung (S. 370) und natürlich die verstärkte Nutzung des Internets (S. 377). Sicherlich sind diese Instrumente geeignet, neue Möglichkeiten auch für Bürger zu schaffen, die sich spontan beteiligen möchten. Doch die genannten Reform-Instrumente würden den Niedergang der Parteien beschleunigen. Auf die Frage, wie diese in ihrer Funktion für das politische System erhalten werden können, haben derzeit weder die Parteien selbst noch die Politikwissenschaft überzeugende konkrete Antworten.

Das Buch ist keine wissenschaftliche Studie, denn weder genügt es in Aufbau und Methodik den entsprechenden Anforderungen, noch hält sich der Verfasser in angemessener Distanz mit eigenen Wertungen zurück. Er gleitet teilweise in pure Polemik ab, wenn er Politiker als ehrgeizige „Egomanen“ bezeichnet (S. 146), Berater in einem missratenen Wortspiel ohne weitere Erläuterung „(Ex)-Politiker, (Ex)-Banker, (Ex)-Perten“ (S. 343) nennt oder zum Abschluss des Buches plötzlich von „Cliquen und Claqueuren“ (S. 435) spricht. Es kommen überdies sehr viele anonyme Quellen („Insider“) zu Wort, Nachweise werden unvollständig geführt (vgl. S. 475), und ausgiebig wird aus nicht-öffentlichen Papieren zitiert - die Nachprüfbarkeit vieler Aussagen ist daher kaum gegeben. Auch ist das Buch nicht nur durchgängig journalistisch flott, sondern wohl auch etwas zu schnell geschrieben worden: Da enden Sätze im Nichts oder sind Umbrüche falsch gesetzt (S. 102, 140, 149), fehlen Buchstaben und ganze Wörter (S. 117, S. 122, S. 142, S. 188) und sind Fachbegriffe schlicht falsch geschrieben (etwa „Refraiming“, S. 148, oder „Dopik“, S. 179); sogar der im Literaturverzeichnis hervorgehobene Verweis auf eine ausführliche InternetBibliographie zum Thema ist grob fehlerhaft. Dennoch kann der Band eine Reihe von Anregungen liefern, und insbesondere die vielfach und ausführlich zitierten internen Dokumente bieten für Forschung und Lehre Einblicke und Ansatzpunkte zum weiteren Nachhaken und Diskutieren.

Sven T. Siefken

\section{Jahrbuch Extremismus und Demokratie: lohnende Lektüre}

Backes, Uwe und Eckhard Jesse (Hrsg.): Jahrbuch Extremismus \& Demokratie 2007, 19. Jahrgang, Nomos Verlagsgesellschaft, Baden-Baden 2008, 532 Seiten, € 49,-.

Mit der einleitenden Bemerkung: „Das neue Jahrtausend hat eine merkliche Verstärkung der zentrifugalen Kräfte des deutschen Parteiensystems gebrach"“, verweisen die Herausgeber des Jahrbuchs Extremismus und Demokratie unmittelbar auf die politische Relevanz der von ihnen nunmehr bereits seit zwei Jahrzehnten zusammengestellten Analysen zu den 